\title{
Brain demyelination due to methionine adenosyltransferase deficiency
}

INSERM

\section{Source}

INSERM. (1999). Orphanet: an online rare disease and orphan drug data base. Brain

demyelination due to methionine adenosyltransferase deficiency. ORPHA:168598

Hypermethioninemia due to methionine adenosyltransferase deficiency is a very rare

metabolic disorder resulting in isolated hepatic hypermethioninemia that is usually benign due to partial inactivation of enzyme activity. Rarely patients have been found to have an odd odor or neurological disorders such as brain demyelination. 